\title{
Foot Scanning in UK, USA and China
}

\author{
Zach ARMITAGE * \\ SATRA Technology, Kettering, UK \\ DOI: $10.15221 / 18.275$ http://dx.doi.org/10.15221/18.275
}

\begin{abstract}
Fit and comfort are almost synonymous in the footwear industry, with sales and brand reputation greatly affected by the fit offered by a product. Additionally, the end consumer is more aware than ever of the effect of poor fit on comfort and foot health.
\end{abstract}

While a degree of discomfort is occasionally accepted in some products, the majority of footwear will not sell if fit is incorrect. The growth of online sales is leading the industry to a greater transparency in fitting guidelines and size marking to reduce returns and secondary purchases intended to assess fit.

A large-scale study of several thousand pairs of feet utilizing 3D scanning technology has produced modern foot data which can lead to the optimisation of fit for a target audience. This can progress to improved population coverage of footwear, increased market potential and augmented comfort levels and general foot health.

Current foot dimension statistics and fitting guidelines are based on data collected a number of decades ago and evidence from this new survey demonstrates that foot shape and size have drifted noticeably over this period. This survey is providing the industry with the required data to evaluate and amend the understanding of foot shape and the dimensions necessary to improve fit.

The statistical evaluation of collected foot data will improve population coverage and enable more informed decisions to be made on fit and sizing to increase comfort and reduce returns.

The scope of this survey also includes the assessment of differences between the three demographics, UK, USA and China. Much of the footwear manufacturing industry has migrated to Asia from Europe and the US while the markets for this footwear remains to the west. This introduces new difficulties in fitting footwear that may have been modelled on Asian feet but intended for a western market.

Similarly, much of the tooling (lasts) used in footwear manufacture were designed in Europe and the US, yet there is an increasingly strong market for European goods in the immerging Eastern markets. Differences in foot shape may require new tooling for such a market.

Keywords: Foot, Foot Scanning, Shoe Size, Fitting, Foot Database, Foot Measurement, 3D Foot

\section{Introduction}

SATRA has studied foot dimensions periodically over the last century with major studies in the 1930's, 1950's and 1980's. The objectives of this latest study were to update the industry guidelines on several measurements vital to good fitting footwear. To understand how the foot is changing. To compare foot dimensions between various demographics including gender, age and global location.

3D scanning technology allows significantly more dimensions to be measured than traditional manual methods. Once the 3D foot file is available any number of measurements can be extracted at a later date. The full survey, SATRA Global Foot Dimensions [1] includes 15 dimensions around the foot and ankle and a further 4 dimensions around the lower leg.

Understanding the shape of the foot and variance in foot dimensions is vital for creating a range of footwear that will offer comfort to a diverse population. Consumers are becoming increasingly aware of fit and comfort and the effects on foot health. Poor fit can lead to a range of foot conditions from blisters, callosities and corns to hallux valgus and bunions.

The growth of online retail has removed the opportunity for in-store fitting, instead multiple sizes are purchased on-line and those not needed are returned at the expense of the retailer. Fitting returns represent a financial burden that can be reduced by clearer online fitting guides, home sizing aids and a greater choice in sizes and fittings to accommodate the wide range of foot shapes.

\section{*Zach.Armitage@SATRA.com}




\subsection{Technology, Accuracy \& Procedure}

\subsection{Accuracy}

3D images were collected using an INFOOT (IFU-H-01) high leg scanner with a scan pitch of $0.5 \mathrm{~mm}$ and manufacturers stated accuracy of $\pm 1 \mathrm{~mm}$ in the $x, y$ and $z$ axes. Foot measurements were taken using INFOOT - Digital Measurement Interface v2.11. Repeated measurements of a homomorphic, resin, foot model was used to confirm correct scanner operation and calculate an uncertainty of measurement. The uncertainty of measurement for both 3D scanning and, for reference, traditional stick and tape foot measuring have been calculated as.

Table 2.1. Uncertainty of Measurement of 3D scanning and traditional foot measurements

\begin{tabular}{|l|cc|}
\hline & $\begin{array}{c}\text { 3D Scanner } \\
(\mathbf{m m})\end{array}$ & $\begin{array}{c}\text { Stick \& Tape } \\
(\mathbf{m m})\end{array}$ \\
\hline \hline Foot Length & \pm 1.89 & \pm 2.15 \\
Joint Girth & \pm 2.02 & \pm 3.34 \\
Instep Girth & \pm 2.41 & \pm 3.27 \\
\hline
\end{tabular}

Stated with a confidence level of $95 \%$, a coverage factor $\mathrm{k}=2$ and an assumed normal distribution.

\subsection{Sample}

Subjects were collected in the UK, USA and China from a range of participating footwear and associated industry companies. A total of approx. 6,240 subjects participated in this study giving a total of 12,474 individual foot scans. The database can be sub-divided into the following demographics:

Table 2.2. Number of foot scans

\begin{tabular}{|c|cc|cc|cc|}
\hline & \multicolumn{2}{|c|}{ UK } & \multicolumn{2}{c|}{ USA } & \multicolumn{2}{c|}{ China } \\
Age & Male & Female & Male & Female & Male & Female \\
\hline \hline $16-25$ & 110 & 462 & 196 & 436 & 1011 & 1486 \\
$26-35$ & 306 & 771 & 652 & 1255 & 1301 & 1539 \\
$36-45$ & 202 & 317 & 513 & 864 & 847 & 1043 \\
$46-55$ & 144 & 204 & 291 & 532 & 345 & 193 \\
$56+$ & 63 & 162 & 135 & 248 & 106 & 75 \\
Total & $\mathbf{8 2 5}$ & $\mathbf{1 , 9 1 6}$ & $\mathbf{1 , 7 8 7}$ & $\mathbf{3 , 3 3 5}$ & $\mathbf{3 , 6 1 0}$ & $\mathbf{4 , 3 3 6}$ \\
\hline
\end{tabular}

Efforts have been made to collect a random sample so as not to introduce bias to the data. The distributions created by the data approximates closely to a normal distribution and the margin of error based on the sample size is less than $1 \mathrm{~mm}$ with a $95 \%$ confidence level.

\subsection{Procedure}

Participants were scanned standing upright with weight evenly distributed over both feet. Scanning was performed throughout the day and each subject was scanned while wearing thin white hose of nominal, un-stretched thickness of $1 \mathrm{~mm}$ that has been demonstrated to have negligible effect on foot dimensions. White hose was used to give a consistent scan surface and to aid in hygiene during the survey.

Each scan was assessed for quality before inclusion in the survey. Any anomalies resulting from scanner or human error, such as light pollution creating superfluous data points, were edited out where possible or the scan removed from the survey. The landmark points located by the software where also validated by a professional and where the software misplaced the landmark points these were corrected. Misplaced landmark points were almost exclusively a problem when locating the metatarsal tibiale and metatarsal fibulare due to the range of foot shapes and bone definition at the joint. 


\section{Foot Measurements}

\subsection{Foot Length}

The linear dimension from the heel (pternion) to furthest toe measured in millimetres through the central axis. The central axis runs from the pternion through the centre point of the joint breadth to the furthest toe, shown in green. The centre point is the point midway between the metatarsal tibiale (first metatarsal head) and metatarsal fibulare (fifth metatarsal head).

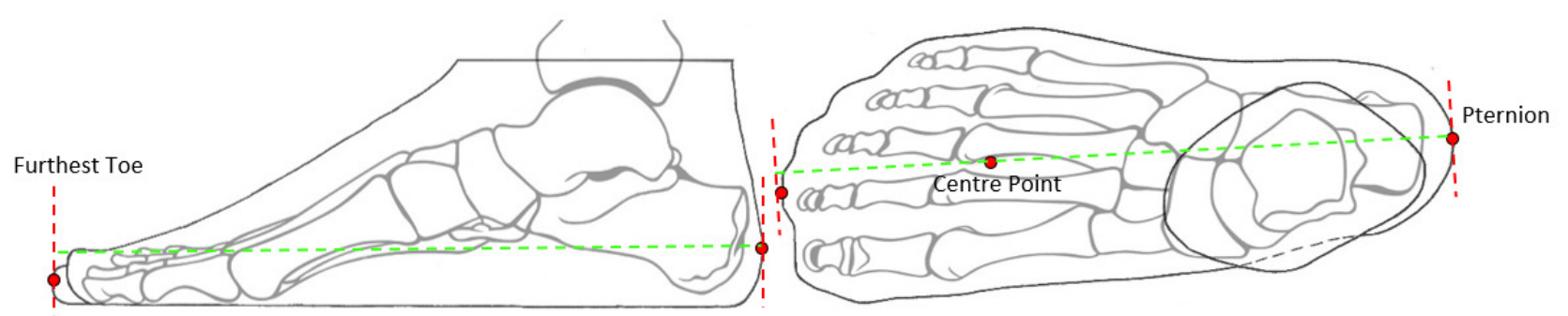

\subsection{Joint Girth}

The circumference around the foot between the metatarsal tibiale and fibulare perpendicular to the standing surface.
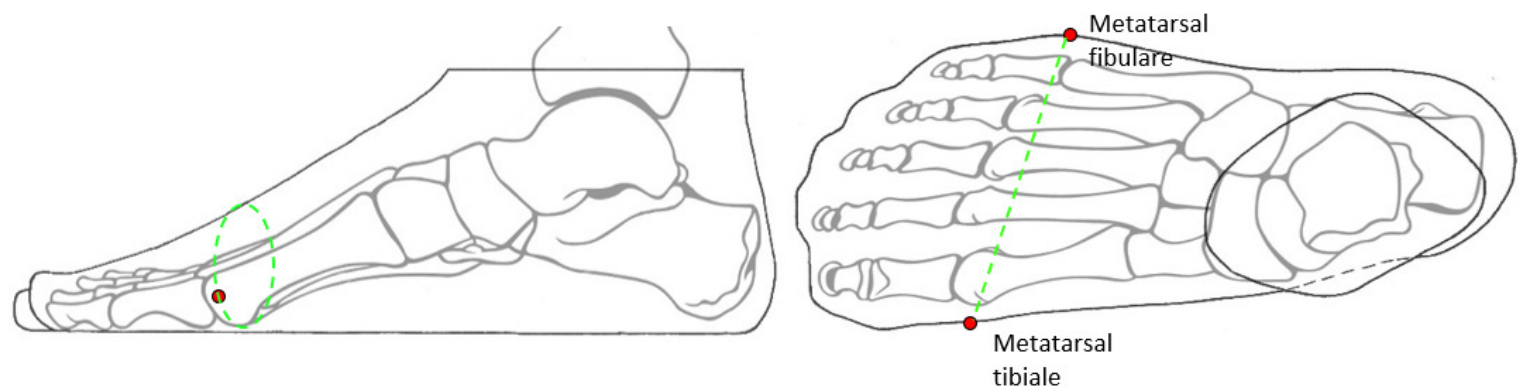

\subsection{Instep Girth}

The circumference around the foot measured at $50 \%$ of the foot length perpendicular to the standing surface.
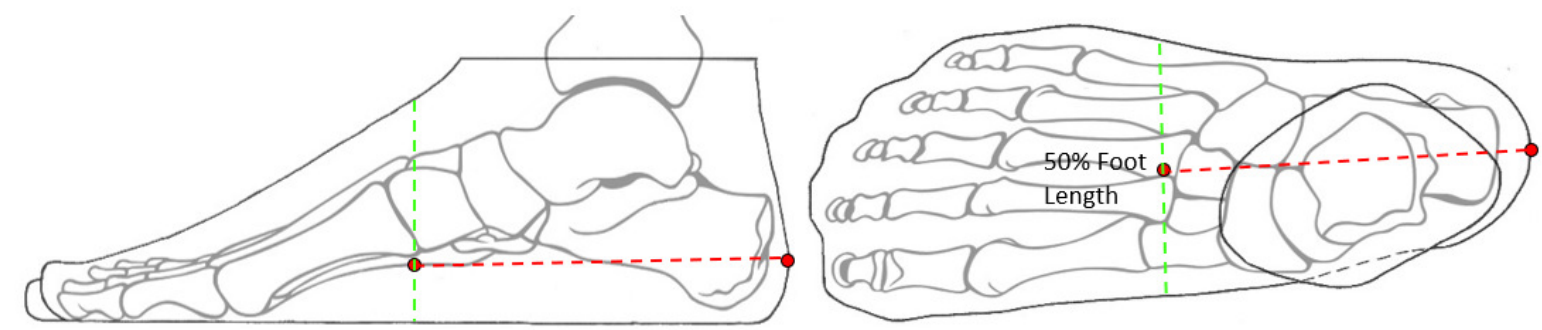

\subsection{Instep Height}

The highest point of the foot measured at $50 \%$ of the foot length perpendicular to the foot axis and standing surface. This generally corresponds with the navicular.
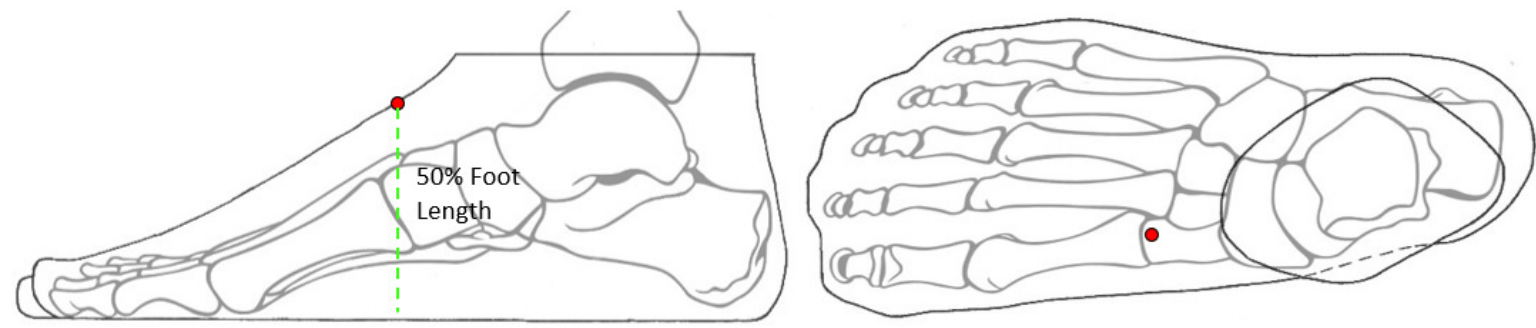


\section{Results}
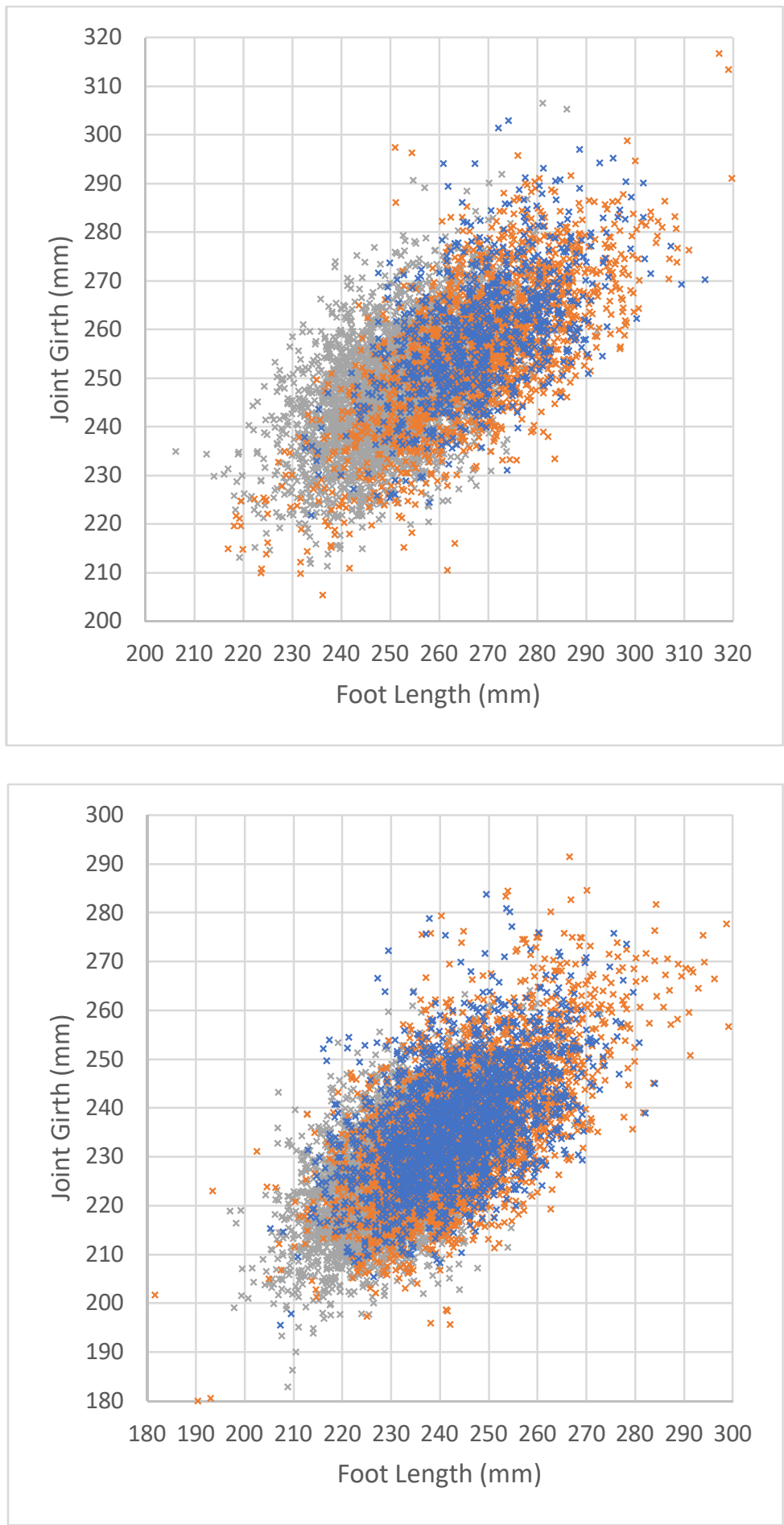

Figure 4.1. Joint girth against foot length for UK (blue), USA (orange) and China (grey) men (top) and women (bottom) 

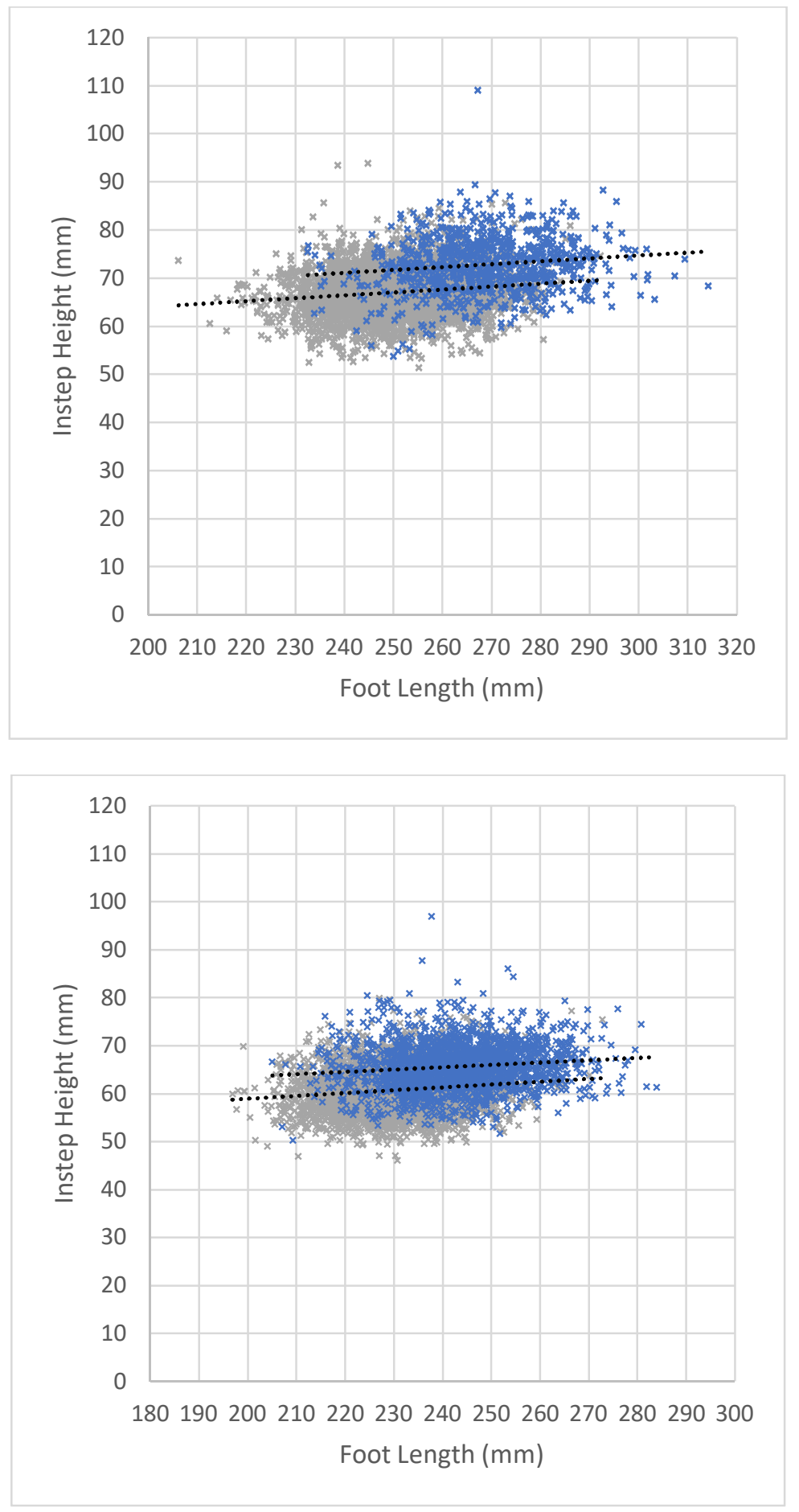

Figure 5.2. Instep height against foot length for UK (blue) and China (grey) men (top) and women (bottom) 


\section{Discussion}

\subsection{Large spread of Joint Girths}

The average spread of $95 \%$ of foot lengths in adults is approximately $49 \mathrm{~mm}$ (equivalent to approx. 6 UK/US shoe sizes or $8 \mathrm{EU}$ sizes). Footwear is sized with respect to foot length and the industry caters well for this variation in foot length. Width fittings are uncommon outside of the USA but the average spread of $95 \%$ of joint girths is approximately $51 \mathrm{~mm}$ (equivalent to approx. $8 \mathrm{UK} / \mathrm{US}$ width fittings or 10 EU width fittings). Only an average of $22 \%$ of adults fall within a standard UK width fitting. A further $38 \%$ have narrow feet and should still attain comfort in a standard fitting, leaving $40 \%$ unaccommodated in standard fittings. Significant population coverage could be achieved by offering 3 width fittings.

\subsection{UK foot dimensions are a close approximation of average European foot dimensions}

A study performed in 2010, Dorothy report [2], averaged a number of foot dimensions from 10,676 feet collected across 11 European countries. The joint girth against foot length and foot breadth against foot length is within approx. $\pm 1 \mathrm{~mm}$ between the Dorothy report and this report [1].

\subsection{Very few average feet}

Approx. $27 \%$ of the sample in this study fall within the average UK shoe size. When taking both foot length and joint girth in to consideration the population that is considered average for both shoe size and width fitting is approx. $7.2 \%$. As further dimensions are considered in defining the average foot the percentage of individuals that fit the average foot is severely diminished. This demonstrates the need for adjustability and a range of choice in fitting of footwear. A conclusion closely matching the results of other biometric studies [2] [3].

\subsection{Chinese instep is lower than the UK}

There is a statistically significant difference between the UK and Chinese foot with regard to the instep height and joint height. The Chinese foot is typically lower than the UK foot which has implications on boot design and shape between these demographics. The difference noted in instep height has a magnitude of one standard deviation between these two groups. The spread of foot height is also noticeably large in all demographics, approx. $30 \mathrm{~mm}$.

\section{References}

[1] Global Foot Dimensions, SATRA Survey 2012:2016, Z Armitage

[2] DOROTHY Mass Foot Measurement Campaign, Aleš Jurca, Tomaž Kolšek, Tina Vidić, UCS

D.O.O., Vrhnika, Slovenia, January 2010

[3] The "Average" Man?, G S Daniels, AD\#010203, Aero Medical Laboratory, December 1952 